country, and to trust for a time entirely to nature. The advice was not agreeable; and she immediately consulted Dr. Bwho, I was informed, not only employed mechanical dilatation to a greater extent, but introduced cutting instruments into the orifice and cervix. Violent mania speedily followed, and her reason has never been perfectly restored. The sterility remains. A similar case of mania has been reported to me, which occurred in Scotland, after the employment of the hyswerotome or some similar weapon."

I am, Sir, your obedient servant,

Savile-row, May 30th, 1865. RoBerT Like, M.D., F.R.S.

\section{THE EPIDEMICS IN THE NORTH OF EUROPE} To the Editor of THE LANckT.

Srk, - Your readers no doubt are tired of the controversy as to the nature of the Dantzic epidemic, though every candid mind will admit that the weapons employed by your correspondent in his letter of the $3 \mathrm{rd}$ inst.- - flippant sarcasm and distortion of facts-are unsuited in the discussion of a practical question. I shall therefore leave him in possession of the field. His well-known desire of notoriety is probably more dear to him than a truthful interpretation of the views of others. I am, Sir, yours faithfully,

Pall-mall, 5th June, 186õ.

$$
\text { A. Tweedie, M.D. }
$$

\section{To the Editor of THe LANCET.}

SrR, - Your last week's journal contains a communication from Dr. Kremiansky giving a description of the post-mortem appearances found in several of the cases of fever which has lately been raging at St. Petersburg. At the first glance of the paper I was led to believe that we had now discovered the connecting link between the cerebro-spinal meningitis and typhus fever; and although this, indeed, may turn out to be the case, yet a more careful perusal of his observations shows that, in the present state of our knowledge, a further obscurity has been thrown on the subject; for it will be seen that the Professor has been describing an hitherto almost unknown pathological condition. He says "the internal surface of the dura mater fon the top and sides of the hemisphere of the brain) is covered here and there with a thin layer of yellowish deposit, which is covered over with light-reddish spots more or less closely grouped together. When washed, a deposit, which consists of tender, villous, dendroid, reddish excrescences swimming under the water, remains on the surface of the dura mater." There is nothing in this description which proves that an inflammatory lymph was present, or that the appearances were due to anything more than an altered condition of exuded blood; as, however, we must not doubt Dr. Kremiansky's opinion that the state he found was due to inflammation, he has brought before our notice a pathological process of an altogether novel character. It may be stated in general terms that a simple idiopathic arachnitis, or inflammation of the serous surfaces of the membranes of the brain, is almost unknown as a post-mortem appearance. What is meant by a meningitis is an inflammation of the pia mater, where the lymph is poured out beneath the arachnoid of the brain and spinal cord-in the so-called subarachnoid space,very little exudation ever transuding through the membrane. In other serous inflammations, as of pericardium, pleura, or peritoneum, the inflammatory products lie between the serous surfaces; but in the case of the nervous centres, owing to their anatomical formation, these products do not occupy a similar position. Thus, in the accounts which we have read of the epidemic cerebro-spinal meningitis, the appearances of the brain and cord, where inflammation was present, corresponded with what is known on the subject; but in Dr. Kremiansky's description we have an altogether different process presented to us. He has described a simple arachnitisan inflammation where the products are interarachnoid, or between the serous surfaces. This is an inflammation having its seat in the dura mater, and which has always been regarded as a condition never arising spontaneously, but due to injury or disease of the bone. It has always been of great practical importance to distinguish a simple arachnitis, or that where the exudation is interarachnoid, as it shows the cause producing it has proceeded from without, and is not a result of idiopathic inflammation. The Professor, therefore, has presented to us a novel pathological process which is of great interest apart from the purpose for which he relates it. It is true that opinions vary as to the source of the membranous layers which are sometimes found on the dura mater in connexion with effusions of blood; some, with Dr. Hodgkin, regarding them as inflammatory ; others, with Prescott Hewett, as mere sanguineous fibrin. The fact, however, remains of such simple form of arachnitis being peculiar and different from what is generally understood by the term meningitis, and what is supposed to exist in the epidemic of the north of Europe.

June, 1865. I am, Sir, your obedient servant

Pathologist.

\section{To the Editor of THE LANCET.}

SIR, - Your latest impression contains a letter from a member of the Pathological Society, regarding which I desire to make a few observations.

I believe there is no candid member of our profession who will deny that Dr. Tweedie's remarks on fever are deserving of the highest respect and consideration. He has for forty years enjoyed rare opportunities, and his fame as a sound pathologist and able physician is founded upon too broad a basis to be disturbed by unfair criticism. I have carefully perused the opinions expressed by Dr. Tweedie in 1829, and also his letter of May 13th in this year, without arriving at your correspondent's conclusions.

Richmond, June, 1865.

I am, Sir, your obedient servant,

R. H.

\section{CONNEXION BETWEEN PHTHISIS AND UTERINE DISEASE. \\ To the Editor of THE LANCET.}

SIR,-In your number for May 27th, Dr. Henry Bennet calls the attention of the profession to the connexion between phthisis and "uterine disease." Without entering into the question whether the phthisis be induced by the depressing effects of the menorrhagia, leucorrhca, \&c., or whether they are merely the common effects of the same constitutional condition, or, in a measure, both united, I think that there is one cause not mentioned which certainly forms the most powerful agent for the production of phthisis in connexion with certain "uterine affections," the consideration of which will help us to explain the nature of that relation in a large class of these cases. But perhaps I may be excused if I take exception to the use of the term "uterine disease," as being scarcely precise enough for the requirements of our study of the subject. The cause above alluded to is that toxæmic state of the blood which so frequently occurs during metritis, and metritic phlebitis after abortion, or particularly after delivery. This is not the place to disenss the nature, origin, \&.., of this blood-state, but I believe it nearly always occurs, more or less, with the above-named diseases.

The train of symptoms are as follows:-First, those of metritis, \&c., with pyrexia; then a cough, slight in some cases, in others severe, becoming acute bronchitis, with or without pneumonia, either general or local, with or without pleuritic effusion. These acute forms are not likely to be overlooked, and, indeed, they have already been alluded to by obstetric authors; and that phthisis should, in strumous constitutions, succeed to this lung irritation, will not be surprising to any one. However, the cases in which these symptoms present, in a minor degree, are not so likely to arrest notice. A little cough, on about the third day after metritic symptoms, must always be viewed with anxiety and suspicion. It may, indeed, be only a common catarrh (to which complaint all the forms are referred by the patients themselves), but the combination should generally be regarded as cause and effect. Nothing, perhaps, best shows this than the length of time required for recovery, and the depressing effect produced by apparently only a few days' general feverishness. In all these forms the reparative powers are at a low ebb. In the best, the uterus but slowly recovers, and chronic metritis, with enlargement, generally succeeds the acute symptoms. Should any lesion have taken place during delivery, it is but slowly repaired, while menorrhagia, leucorrhoa, \&c., are generally present. Should cellulitis have occurred, the effusion is slowly absorbed. Meanwhile the lungs slowly improve, and at lastsometimes after two or three months in moderate casesrecovery takes place. Should, on the contrary, the constitution have been previously depressed, or of the strumous type, or the depression by the toxæmia have been very great, espe- 